\title{
Expression and significance of c-kit and epithelial-mesenchymal transition (EMT) molecules in thymic epithelial tumors (TETs)
}

\author{
Zhigang Wü, Songtao Xue", Bin Zheng", Rongjin Ye, Guobing Xu, Shuliang Zhang, Taidui Zeng, \\ Wei Zheng, Chun Chen
}

Thoracic Department, Fujian Medical University Union Hospital, Fuzhou 350001, China

Contributions: (I) Conception and design: W Zheng, C Chen; (II) Administrative support: W Zheng, C Chen; (III) Provision of study materials or patients: Z Wu, S Xue, B Zheng; (IV) Collection and assembly of data: R Ye, G Xu, S Zhang, T Zeng; (V) Data analysis and interpretation: R Ye, G $\mathrm{Xu}$, S Zhang, T Zeng; (VI) Manuscript writing: All authors; (VII) Final approval of manuscript: All authors.

"These authors contributed equally to the study, and should be considered as co-first authors.

Correspondence to: Wei Zheng. Thoracic Department, Fujian Medical University Hospital, No. 29, Xinquan Road, Fuzhou 350001, China.

Email: lacustrian@163.com.

Background: To investigate the expression and significance of c-kit and epithelial-mesenchymal transition (EMT) molecules (E-cadherin, N-cadherin, Twist, Snail) in thymic epithelial tumors (TETs).

Methods: The tissue microarray technology and immunohistochemistry MaxVisionTM-use kit were used to detect the expression of c-kit and EMT molecular markers in 150 cases of paraffin sections of TET tissue and analysis the correlation between c-kit and EMT molecules and explore the malignancy and the relationship of clinicopathological parameters between c-kit, EMT molecules and TETs.

Results: The expression difference of c-kit and EMT molecular markers (E-cadherin, N-cadherin, Snail, Twist) in TETs subtypes was statistically significant $(\mathrm{P}<0.01)$ and their positive expression rate of thymic carcinoma was significantly higher than that in thymoma, and the difference was statistically significant, respectively $(\mathrm{P}<0.01)$. There is a negative correlation between the expression of c-kit and E-cadherin as well as a positive correlation between the expression level of c-kit, $\mathrm{N}$-cadherin, Twist, and Snail. Furthermore, E-cadherin was negatively correlated with $\mathrm{N}$-cadherin, Twist, and Snail while N-cadherin expression was positively correlated with Twist, Snail.

Conclusions: Five indicators (c-kit, E-cadherin, N-cadherin, Twist, and Snail) may determine the malignancy of TETs, especially for distinguishing thymoma and thymic carcinoma.

Keywords: c-kit; EMT; Snail; thymic epithelial tumors (TETs); Twist

Submitted Sep 05, 2019. Accepted for publication Oct 21, 2019.

doi: $10.21037 /$ jtd.2019.10.56

View this article at: http://dx.doi.org/10.21037/jtd.2019.10.56

\section{Introduction}

Thymic epithelial tumors (TETs) originate from thymic epithelial cells, and mainly include tumors with different biological functions and pathological types such as thymoma, thymic carcinoma, and thymic carcinoid. TETs are the most common tumors in the anterior mediastinum, but the low incidence in the population makes it difficult to collect large-scale cases for analysis, which limits the research progress of this disease. There are several subtypes of TETs, which are classified into non-invasive, invasive, and malignant according to the degree of malignancy. The World Health Organization (WHO classification, 2015) divided thymoma into six subtypes: thymoma $\mathrm{A}, \mathrm{AB}, \mathrm{B} 1$, B2, B3, and thymic carcinoma (C). TETs are different from other tumors in that their benign and malignant properties cannot be completely determined according to the corresponding histology $(1,2)$. Surgery is the main treatment, but there is still no agreement on the treatment of patients with advanced unresectable, metastatic, and 
relapsed refractory patients. The prognosis of the patients is determined by the pathological type and effect of surgical resection. But until then, the pathogenesis of TETs remains unclear. Recently, many studies have shown that c-kit is closely related to the invasion and metastasis of some tumor cells, while epithelial-mesenchymal transition (EMT) is a key factor associated with tumor progression, tumor cell invasion, and distant metastasis.

As an oncogene found on chromosome 4q11-12, c-kit, also known as the stem cell factor receptor named CD117, was first discovered in HZ4 cat sarcoma virus in $1986(3,4)$. Stem cell factors can bind to a c-kit receptor to form a receptor-dimer complex, which induces molecular phosphorylation and activate downstream signaling pathways (such as PI3K pathway, JAK-STAT pathway, RAS-ERK pathway, SFK pathway) to regulate cell proliferation, differentiation, adhesion, and apoptosis. The survival, differentiation, proliferation, and growth of hematopoietic cells require the regulation of stem cell factor (SCF), which needs to be combined with the c-kit receptor. Studies have found that the differentiation and proliferation abnormalities of different cells in the human body are closely related to the abnormal activation of c-kit/SCF signaling system, so the function loss of c-kit receptor and functional activation are closely related to the occurrence of many malignant tumors (5-7). Given this, the drug intervention in the function of c-kit receptor and the inhibition of the abnormal downstream signaling may provide new approaches for the treatment of tumors. Some studies have found that c-kit is overexpressed in many human malignancies, including gastrointestinal stromal tumors (GISTs), chronic myeloid leukemia, mastocytoma, melanoma, and seminoma (8). Overexpression of c-kit protein is closely related to mutation of the c-kit gene $(9,10)$, which leads to functional activation of c-kit receptor through c-kit gene mutation, triggering downstream abnormal signal transduction and abnormal proliferation of tumor cells. As a useful immunohistochemical marker that combined with CD5 immunohistochemistry, c-kit is important for the differential diagnosis of primary thymic carcinoma and lung squamous cell carcinoma (11). It has been reported that the expression ratio of c-kit in thymic carcinoma range from $50 \%$ to $88 \%$, but rarely expressed (0-5\%) in thymoma $(11,12)$. However, the special mechanism of abnormal expression of c-kit and the development of TETs remain to be further studied.

EMT refers to the transformation of epithelial phenotype to the interstitial phenotype, which is closely related to the invasiveness of tumor cells. It was first proposed by Greenburg and Hay in 1982 (13). EMT often occurs in dedifferentiated cancer cells at the edge of cancerous tissues and has been proved in a variety of cancer tissues (e.g., thyroid, breast, prostate, etc.) $(14,15)$. At the molecular level, EMT represents the absence of an epithelial phenotype and the cellular properties of the interstitial phenotype, enhancing cell metastasis and invasiveness (16). This process involves the loss of cell viscosities, the fundamental reorganization of the cytoskeleton, which endows polarity to the basal cells and is also associated with cell infiltration properties obtained through secretion of soluble proteases and resistance to aging and apoptosis (17). EMT is a pathway with multiple regulatory controls. The enhancement of the signal activity of TGF- $\beta$ can be seen when EMT occurs (such as organ formation, inflammation, and tumor infiltration) $(18,19)$. Typical TGF- $\beta$ signal activates the Smad family transcription factor by binding to its corresponding cell surface receptor, followed by translocation to the nucleus and combine with the "EMT regulatory gene" (Snail and Twist family transcription factors) $(20,21)$. Non-Smad signaling molecules acting on downstream TGF- $\beta$ promote the development of EMT processes, including activation of Rho GTPase, PI3K, and MAPK (17). Taken together, these effects mediate transcriptional repression of genes associated with cell polarity and cell-cell adhesion such as Rho A and E-cadherin $(22,23)$.

The cadherin superfamily includes E-cadherin (E-cad), $\mathrm{N}$-cadherin $(\mathrm{N}$-cad) and placental cadherin (P-cadherin), which are required for $\mathrm{Ca}^{2+}$. The E-cadherin coding gene is located on chromosome 16q22.1. The absence of adhesion ability of E-cadherin's is the key for cell invasiveness. The down-regulation or deletion of E-cadherin expression ultimately leads to a decrease in the stability of epithelial cells and disrupts cell polarity. The non-invasive cell surface expresses more E-cadherin molecules, and the antiE-cadherin antibody can block the connection between cells so that the cells can obtain high invasiveness against collagen fibers (24). After transfection of E-cadherin cDNA, tumorigenicity and metastasis were inhibited in cells with the high metastatic ability and low expression of E-cadherin. Transfection of E-cad antisense RNA into E-cadherin high-expression tumor cell lines can significantly improve the metastatic potential, suggesting that E-cadherin can inhibit tumor metastasis (25). A significant correlation was found between the low expression of E-cadherin and the invasive ability of colorectal cancer. The expression level of E-cadherin was significantly decreased in 2 of 7 colorectal 
cancer cell lines. Further in vitro invasion experiments revealed that these two colorectal cancer cell lines could infiltrate collagen gel and collagen membrane $(26,27)$. Therefore, down-regulation of E-cadherin is considered to be a marker of EMT development.

The N-cadherin-encoding gene is located on chromosome 18q11.2. The encoded protein has a molecular mass of $127 \mathrm{kD}$ and is expressed only in the neuroectoderm and mesoderm, such as mature muscle, nerve, and hematopoietic tissue but is rarely expressed in normal epithelial cells. The expression of $\mathrm{N}$-cad in epithelial tissues can alter the normal morphological and biological behavior of epithelial cells, transforming epithelial cells into mesenchymal cells (EMT). In vitro studies have shown the significant enhancement of invasion in tumor cells transfected with $\mathrm{N}$-cadherin $(28,29)$. Hazan et al. found that anti-intravascular $\mathrm{N}$-cadherin antibody can effectively reduce the metastasis of breast cancer cells. Compared with the low expression of E-cadherin, the high expression of $\mathrm{N}$-cadherin can directly promote the progression of EMT (28). Previous studies have reported that $\mathrm{N}$-cadherin can help tumor cells escape immune rejection and promote tumor cell adhesion to extracellular matrix and endothelium, playing an important role in tumor cell invasion and metastasis (30). Gwak et al. found that $\mathrm{N}$-cadherin can help tumor cells resist apoptosis and thus participate in tumor progression (31). All studies have shown that $\mathrm{N}$-cadherin is closely related to tumor invasion and metastasis.

Twist also known as Twist1, is an oncogene closely related to EMT and shares extensive homology with the Twist-associated protein (Twist2), which is critical for neural tube formation and is involved in the regulation of chondrocyte differentiation in skeletal development and transformation (32). Abnormal expression or methylation of Twist is often found in metastatic tumors. In 2004, Yang first identified Twist as a factor that promotes tumor metastasis in a mouse cogenetic breast cancer cell line $(33,34)$. Sahlin pointed out that Twist is a susceptibility gene for breast cancer (35). Overexpression of Twist is also associated with the development of many other tumors, such as prostate cancer, ovarian epithelial cancer, transitional cell carcinoma, bladder cancer, stomach cancer, colorectal cancer and thyroid cancer (36).

Snail is a transcription factor containing a zinc finger structure, first discovered in Drosophila, and is a Drosophila embryonic protein. Snail is essential for normal human development, such as the formation of mesoderm and neural crest, and the development of the central nervous system. The Snail superfamily includes Snail1, Snail2 (also known as Slug) and all family members have highly conserved carboxy-terminal domains that can be linked to the specific DNA sequence of E-box in the E-cadherin promoter (37). Overexpression of Snail is associated with a variety of malignancies, such as breast cancer, ovarian cancer, gastric cancer, hepatocellular carcinoma, and colon cancer (38).

Until now, EMT has become a hotspot in cancer research, and there is a growing body of data showing that EMT plays a key role in cancer metastasis. Snail and Twist are transcriptional regulators that promote EMT mainly through down-regulate the transformation of E-cadherin into $\mathrm{N}$-cadherin. Therefore, we designed to investigate whether c-kit expression is related to EMT by validating the expression of c-kit, E-cadherin, $\mathrm{N}$-cadherin, Snail and Twist in TETs and the association between c-kit expression level and EMT and explore whether there is a difference in different subtypes of TETs, and then provide directions for exploring effective targeted therapy for invasive and malignant thymic tumors.

\section{Methods}

\section{Patients and tissue collection}

A total of 150 specimens analyzed in this study were obtained from the Department of Thoracic Surgery, Fujian Medical University Union Hospital from January 2007 to December 2013. The clinical-pathological data were completed, and all cases, including 78 males and 72 females, were performed surgery without chemotherapy, radiotherapy, or biological therapy. The age ranges from 10 to 82 years old, and the median age was 46 years old. According to the WHO standard classification: 11 cases were typed A, 35 cases were type $A B, 19$ cases were type B1, 40 cases were type B2, 23 cases were type B3, and 22 cases were type $\mathrm{C}$. All specimens were fixed in formalin and embedded in paraffin. All specimens were made into tissue arrays, and $4 \mu \mathrm{m}$ serial sections were used to obtain tissue microarray for immunohistochemistry experiments. All patients gave their informed consent before their inclusion in the study. The study was approved by the institutional research ethics committee.

\section{Western blot analysis}

Western blot analysis was performed in $12 \%$ SDS-PAGE gels electrophoresis under denaturing and reducing conditions. Specific primary antibodies for CD117 (RMA- 

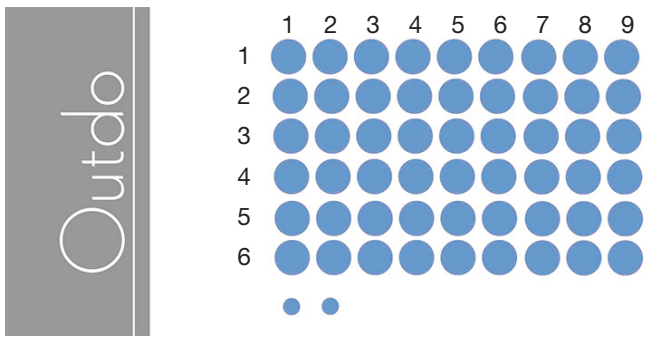

Figure $16 \times 9$ array arrangement was used to arrange 150 thymic epithelial tumor wax specimens.

0632, Maxim, China), E-cadherin (MAB-0589, Maxim, China), N-Cadherin (MAB-0571, Maxim, China), Snail (HZ92811, Qiagen, Shanghai), Twist (HZ38821, Qiagen, Shanghai) were used to detect the protein expression of these genes.

\section{Tissue array manufacture}

One hundred fifty TET wax specimens were designed into a $6 \times 9$ array arrangement (Figure 1). The wax block sections were taken for immunohistochemical staining. A blank wax block was prepared as the acceptor wax block and heated at $46^{\circ} \mathrm{C}$ in a water bath for about 3 minutes, and then a $6 \times 9$ tissue receptor hole was punched in the acceptor wax block by using a self-made tissue array device. The labeled tumor tissue was drilled from the donor wax block and transferred to the acceptor wax block. Two tumor tissue columns were drilled from each donor wax block. And then the acceptor wax block was placed in a $53{ }^{\circ} \mathrm{C}$ oven to fuse wax block with tissue core and cool down naturally. The tissue was cut continuously for $4 \mu \mathrm{m}$, and the poly-lysine gel slides were taken into a tissue array and baked in an oven at $60^{\circ} \mathrm{C}$ for $2 \mathrm{~h}$.

\section{Immunobistochemistry}

Serially sectioned specimen wax pieces at $4 \mu \mathrm{m}$ and treated with poly-lysine slides and baked at $60^{\circ} \mathrm{C}$ in the oven for $2 \mathrm{~h}$. Soak the sheets in xylene I and xylene II for $15 \mathrm{~min}$ and washing for $5 \mathrm{~min}$ in anhydrous, $95 \%, 85 \%$, and $70 \%$ gradient alcohol, respectively, and then washed with PBS 3 times for $2 \mathrm{~min}$. The tissue antigen is repaired according to the requirements of each antibody (e.g., citric acid high pressure for $1.5 \mathrm{~min}$ ). One drop or $50 \mu \mathrm{L}$ of $3 \%$ hydrogen peroxide was added to each section and incubated at room temperature for 10 minutes to block the activity of endogenous peroxidase. Adding 1 drop or $50 \mu \mathrm{L}$ of non- immune goat serum to each section and incubate for 10 minutes at room temperature. Adding $50 \mu \mathrm{L}$ of the primary antibody to each section and incubate for 60 minutes at room temperature. $50 \mu \mathrm{L}$ of the ready-to-use MaxVision ${ }^{\text {TM }}$ (KIT-5910 MaxVision ${ }^{\text {TM }}$ HRP-Polymer antirabbit/mouse IHC Kit) reagent was added and incubated for 15 minutes at room temperature. Adding $100 \mu \mathrm{L}$ of freshly prepared $\mathrm{DAB}$ coloring solution for 5 minutes and observe under a microscope. Rinsed with water and counterstained with hematoxylin, gradient dehydrated with alcohol and hyalinized with xylene, and then sealed with neutral gum. PBS was used as a negative control, and a mouse lung cancer tissue positive slice was taken as a positive control.

\section{Interpretation of the IHC results}

Two experienced pathologists were invited to observe the results through a double-blind reading mode. The c-kit protein is mainly located in the cell membrane and cytoplasm. Therefore, the cell membrane and cytoplasm showed light yellow or brownish yellow were taken as positive cells. Five high power fields $(\times 400)$ were randomly selected from each array under the microscope, and the percentage of positive cells was recorded. The interpretation criteria were as following: $0 \%$ for $(-)$, $1-10 \%$ for $(+), 10-50 \%$ for $(++)$, and $51-100 \%$ for $(+++)$. $\mathrm{E}$-cadherin and $\mathrm{N}$-cadherin are mainly located in the cell membrane, and partially located in the cytoplasm. Therefore, the cell membrane or cytoplasm showed brownyellow particles were taken as positive cells. Five highpower fields $(\times 400)$ are randomly selected from each array under the microscope, and the percentage of positive cells was recorded. The following criteria were used: E-cadherin: $\geq 70 \%$ for $(+)$ and $<70 \%$ for (-), N-cadherin: $\geq 5 \%$ for $(+)$ and $<5 \%$ for $(-)$. Twist and Snail are mainly located in the nucleus and cytoplasm, brown-yellow cells that observed in the nucleus and cytoplasm were treated as positive cells. Five high power fields $(\times 400)$ were randomly selected for each array under the microscope, and 200 cells were counted for each field. The following criteria were used: $<10 \%$ for positive cells $(-),(+)$ for positive cells between $10 \%$ and $25 \%,(++)$ for positive cells at $26-50 \%$, positive cell rate $>50 \%$ was $(+++)$. Both $(+),(++)$, and $(+++)$ were judged as statistical positive.

\section{Statistical analysis}

Statistical analysis was performed using SPSS 24.0 statistical 

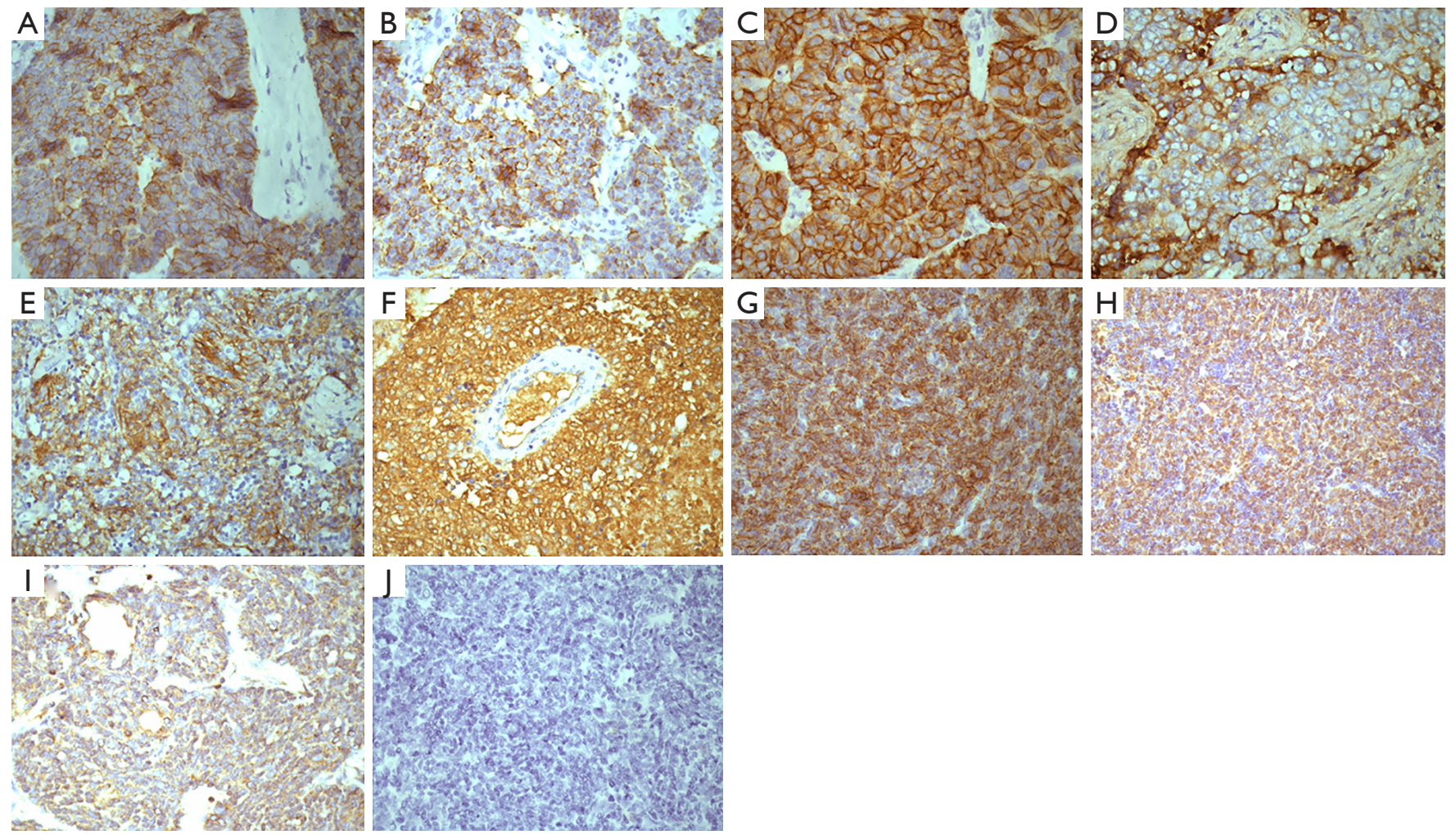

Figure 2 Demonstrative IHC staining of c-kit (A,B,C), E-cad (D,E,F), N-cad (G), Twist (H), and Snail (I) in different type thymic tumors. (A) Positive expression of c-kit in type $\mathrm{C}$ thymic tumor; (B) positive expression of c-kit in type B2 thymic tumor; (C) positive expression of c-kit in type B3 thymic tumor; (D) positive expression of E-cad in type A thymic tumor; (E) positive expression of E-cad in type B3 thymic tumor; (F) Positive expression of E-cad in type AB thymic tumor; (G) positive expression of $\mathrm{N}$-cad in type $\mathrm{C}$ thymic tumor; (H) positive expression of Twist in type $\mathrm{C}$ thymic tumor; (I) positive expression of Snail in type $\mathrm{C}$ thymic tumor; $(\mathrm{J})$ no stained thymic tumor. (Magnification $\times 400$ ).

software, with $\mathrm{P}<0.05$ as a statistically significant difference. The relationship between the expression of c-kit and EMT molecules and clinicopathological parameters was determined by $\chi^{2}$ test and Fisher's exact probability method, the correlation between c-kit and EMT expression was analyzed by Spearman correlation.

\section{Results}

\section{Expression of c-kit and EMT molecules was upregulated in TETs}

The positive c-kit expression showed light yellow or brownish yellow, mainly located in the cell membrane or cytoplasm, and was not expressed in normal thymus tissue. The positive expression rate of c-kit in TETs was $14.67 \%$ (22/150), and the positive expression rates of each subtype were as followings: type A $(9.09 \%, 1 / 11)$, type $\mathrm{AB}(2.86 \%$,
1/35), B1 type (5.26\%, 1/19), B2 type (5\%, 2/40), B3 type (8.70\%, 2/23), Type C $(68.18 \%, 15 / 22)$. The positive expression of c-kit was statistically significant among the subtypes of TETs $(\mathrm{P}<0.01$, Figure $2 A, B, C$, Table 1$)$. The positive expression rate of c-kit in thymic carcinoma was $68.18 \%(15 / 22)$, which was significantly higher than that of thymoma $(5.47 \%, 7 / 128)$ and was statistically significant $(\mathrm{P}<0.01$, Table 2).

The positive expression products of E-cad were brownish yellow, mainly located in the cell membrane or cytoplasm, and positively expressed in normal thymus tissue. The positive expression rate of E-cad in TETs was $68.67 \%(103 / 150)$, and the positive expression rates of each subtype were as followings: type $\mathrm{A}(81.82 \%, 9 / 11)$, type $\mathrm{AB}$ $(85.71 \%, 30 / 35)$, B1 type $(78.95 \%, 15 / 19)$, B2 type $(80 \%$, $32 / 40)$, B3 type $(73.91 \%, 17 / 23)$, Type C $(22.73 \%, 5 / 22)$. The positive expression of E-cad in the subtypes of TETs was statistically significant $(\mathrm{P}<0.01$, Figure $2 D, E, F$, Table 3$)$. 
Table 1 Expression of c-kit in thymic epithelial tumors

\begin{tabular}{|c|c|c|c|c|c|c|}
\hline \multirow{2}{*}{$\begin{array}{l}\text { WHO } \\
\text { classification }\end{array}$} & \multirow[b]{2}{*}{ Cases } & \multicolumn{3}{|c|}{ c-kit } & \multirow[b]{2}{*}{$\chi^{2}$} & \multirow[b]{2}{*}{$\mathrm{P}$} \\
\hline & & + & - & $\begin{array}{l}\text { Positive } \\
\text { rate (\%) }\end{array}$ & & \\
\hline A & 11 & 1 & 10 & 9.09 & 41.412 & $<0.01$ \\
\hline$A B$ & 35 & 1 & 34 & 2.86 & & \\
\hline B1 & 19 & 1 & 18 & 5.26 & & \\
\hline B2 & 40 & 2 & 38 & 5.00 & & \\
\hline B3 & 23 & 2 & 21 & 8.70 & & \\
\hline C & 22 & 15 & 7 & 68.18 & & \\
\hline
\end{tabular}

Table 2 Differential expression of c-kit in thymoma and thymic carcinoma

\begin{tabular}{|c|c|c|c|c|}
\hline \multirow{2}{*}{ Classification } & \multicolumn{2}{|c|}{ c-kit } & \multirow{2}{*}{$\chi^{2}$} & \multirow{2}{*}{$\mathrm{P}$} \\
\hline & + & - & & \\
\hline Thymoma & 7 & 121 & 58.994 & $<0.01$ \\
\hline Thymic carcinoma & 15 & 7 & & \\
\hline
\end{tabular}

Table 3 Expression of E-cad in thymic epithelial tumors

\begin{tabular}{|c|c|c|c|c|c|c|}
\hline \multirow{2}{*}{$\begin{array}{l}\text { WHO } \\
\text { classification }\end{array}$} & \multirow[b]{2}{*}{ Cases } & \multicolumn{3}{|c|}{ E-cad } & \multirow[b]{2}{*}{$\chi^{2}$} & \multirow[b]{2}{*}{$\mathrm{P}$} \\
\hline & & + & - & $\begin{array}{c}\text { Positive rate } \\
\text { (\%) }\end{array}$ & & \\
\hline A & 11 & 9 & 2 & 81.81 & 28.011 & $<0.01$ \\
\hline$A B$ & 35 & 30 & 5 & 85.71 & & \\
\hline B1 & 19 & 15 & 4 & 78.95 & & \\
\hline B2 & 40 & 32 & 8 & 80.00 & & \\
\hline B3 & 23 & 17 & 6 & 73.91 & & \\
\hline C & 22 & 5 & 17 & 22.73 & & \\
\hline
\end{tabular}

Table 4 Differential expression of E-cad in thymoma and thymic carcinoma

\begin{tabular}{|c|c|c|c|c|}
\hline \multirow{2}{*}{ Classification } & \multicolumn{2}{|c|}{ E-cad } & \multirow{2}{*}{$\chi^{2}$} & \multirow{2}{*}{$P$} \\
\hline & + & - & & \\
\hline Thymoma & 103 & 25 & 31.048 & $<0.01$ \\
\hline $\begin{array}{l}\text { Thymic } \\
\text { carcinoma }\end{array}$ & 5 & 17 & & \\
\hline
\end{tabular}

Table 5 Expression of $\mathrm{N}$-cad in thymic epithelial tumors

\begin{tabular}{|c|c|c|c|c|c|c|}
\hline \multirow{2}{*}{$\begin{array}{l}\text { WHO } \\
\text { classification }\end{array}$} & \multirow[b]{2}{*}{ Cases } & \multicolumn{3}{|c|}{$\mathrm{N}$-cad } & \multirow[b]{2}{*}{$\chi^{2}$} & \multirow[b]{2}{*}{$\mathrm{P}$} \\
\hline & & + & - & $\begin{array}{l}\text { Positive } \\
\text { rate (\%) }\end{array}$ & & \\
\hline A & 11 & 0 & 11 & 0.00 & 41.736 & $<0.01$ \\
\hline$A B$ & 35 & 0 & 35 & 0.00 & & \\
\hline B1 & 19 & 1 & 18 & 5.26 & & \\
\hline B2 & 40 & 2 & 38 & 5.00 & & \\
\hline B3 & 23 & 2 & 21 & 8.70 & & \\
\hline C & 22 & 14 & 8 & 63.64 & & \\
\hline
\end{tabular}

Table 6 Differential expression of N-cad in thymoma and thymic carcinoma

\begin{tabular}{lrrrr}
\hline \multirow{2}{*}{ Classification } & \multicolumn{2}{c}{ N-cad } & \multirow{2}{*}{$\chi^{2}$} & $\mathrm{P}$ \\
\cline { 2 - 3 } & \multicolumn{1}{c}{5} & - & & \\
\hline Thymoma & 5 & 123 & 60.546 & $<0.01$ \\
Thymic carcinoma & 14 & 8 & & \\
\hline
\end{tabular}

The positive expression rate of E-cad in thymoma was $80.47 \%(103 / 128)$, which was significantly higher than that in thymic carcinoma $(22.73 \%, 5 / 22)$ and was statistically significant $(\mathrm{P}<0.01$, Table 4).

The positive expression products of $\mathrm{N}$-cad are brownish yellow, found in the cell membrane or cytoplasm, and no positive expression was observed in normal thymus tissue. The positive expression rate of $\mathrm{N}$-cad in TETs was $(12.67 \%$, 19/150), and the positive expression rates of each subtype were: type $\mathrm{A}(0 \%, 0 / 11)$, type $\mathrm{AB}(0 \%, 0 / 35), \mathrm{B} 1$ type (5.26\%, 1/19), B2 type (5\%, 2/40), B3 type (8.70\%, 2/23), Type C $(63.64 \%, 14 / 22)$. The positive expression of $\mathrm{N}-\mathrm{cad}$ was significantly different between the subtypes of TETs $(\mathrm{P}<0.01$, Figure $2 G$, Table 5). The positive expression rate of $\mathrm{N}$-cad in thymic carcinoma was $(63.64 \%, 14 / 22)$, which was significantly higher than $3.91 \%(5 / 128)$ in thymoma with statistical significance $(\mathrm{P}<0.01$, Table 6).

The positive expression of Twist was brownish yellow, mainly located in the nucleus or cytoplasm, and was not expressed in normal thymus tissue. The positive expression rate of Twist in TETs was 26.67\% (40/150), and the positive expression rates of each subtype were: type A (18.18\%, 
Table 7 Expression of Twist in thymic epithelial tumors

\begin{tabular}{|c|c|c|c|c|c|c|}
\hline \multirow[b]{2}{*}{$\begin{array}{l}\text { WHO } \\
\text { classification }\end{array}$} & \multirow[b]{2}{*}{ Cases } & \multicolumn{3}{|c|}{ Twist } & \multirow[b]{2}{*}{$\chi^{2}$} & \multirow[b]{2}{*}{$\mathrm{P}$} \\
\hline & & + & - & $\begin{array}{l}\text { Positive } \\
\text { rate (\%) }\end{array}$ & & \\
\hline A & 11 & 2 & 9 & 18.18 & 24.397 & $<0.01$ \\
\hline$A B$ & 35 & 5 & 30 & 14.29 & & \\
\hline B1 & 19 & 4 & 15 & 21.05 & & \\
\hline B2 & 40 & 8 & 32 & 20.00 & & \\
\hline B3 & 23 & 5 & 18 & 21.74 & & \\
\hline C & 22 & 16 & 6 & 72.73 & & \\
\hline
\end{tabular}

Table 8 Differential expression of Twist in thymoma and thymic carcinoma

\begin{tabular}{|c|c|c|c|c|}
\hline \multirow{2}{*}{ Classification } & \multicolumn{2}{|c|}{ Twist } & \multirow{2}{*}{$\chi^{2}$} & \multirow{2}{*}{$\mathrm{P}$} \\
\hline & + & - & & \\
\hline Thymoma & 24 & 104 & 27.970 & $<0.01$ \\
\hline Thymic carcinoma & 16 & 6 & & \\
\hline
\end{tabular}

Table 9 Expression of Snail in thymic epithelial tumors

\begin{tabular}{lrrrrrr}
\hline \multirow{2}{*}{$\begin{array}{l}\text { WHO } \\
\text { classification }\end{array}$} & Cases & \multicolumn{5}{c}{ Snail } \\
\cline { 3 - 5 } & & + & $-\begin{array}{r}\text { Positive } \\
\text { rate (\%) }\end{array}$ & & P \\
\hline A & 11 & 1 & 10 & 9.09 & 37.048 & $<0.01$ \\
AB & 35 & 3 & 32 & 8.57 & & \\
B1 & 19 & 3 & 16 & 15.79 & & \\
B2 & 40 & 5 & 35 & 12.50 & & \\
B3 & 23 & 5 & 18 & 21.74 & & \\
C & 22 & 17 & 5 & 77.27 & & \\
\hline
\end{tabular}

Table 10 Differential expression of Snail in thymoma and thymic carcinoma

\begin{tabular}{lrrrrr}
\hline \multirow{2}{*}{ Classification } & \multicolumn{2}{c}{ Snail } & \multirow{2}{*}{$\chi^{2}$} & $\mathrm{P}$ \\
\cline { 2 - 3 } & \multicolumn{1}{c}{+} & \multicolumn{1}{c}{$\begin{array}{l}\text { 17 } \\
\text { Thymoma }\end{array}$} & & & \\
\hline Thymic carcinoma & 17 & 5 & & \\
\hline
\end{tabular}

2/11), type $\mathrm{AB}(14.29 \%, 5 / 35)$. B1 type (21.05\%, 4/19), B2 type $(20 \%, 8 / 40)$, B3 type $(21.74 \%, 5 / 23)$, Type C $(72.73 \%$, 16/22). The positive expression of Twist in the subtypes of TETs was statistically significant $(\mathrm{P}<0.01$, Figure $2 \mathrm{H}$, Table 7). The positive expression rate of Twist in thymic carcinoma was $72.73 \%(16 / 22)$ higher than that in thymoma $(18.75 \%, 24 / 128)$ and was statistically significant $(\mathrm{P}<0.01$, Table 8).

The positive expression products of Snail were light yellow or brownish yellow, mainly located in the nucleus or cytoplasm, and has no positive expression in normal thymus tissue. The positive expression rate of Snail in TETs was $22.67 \%(34 / 150)$, and the positive expression rates of each subtype were: type A $(9.09 \%, 1 / 11)$, type $\mathrm{AB}(8.57 \%, 3 / 35)$. B1 type (15.79\%, 3/19), B2 type (12.50\%, 5/40), B3 type (21.74\%, 5/23), Type C $(77.27 \%, 17 / 22)$. The positive expression of Snail was significantly different between the subtypes of TETs $(\mathrm{P}<0.01$, Figure 2I, Table 9). The positive expression rate of Snail in thymic carcinoma was $77.27 \%(17 / 22)$, which was significantly higher than that of thymoma $(13.28 \%, 17 / 128)$ with statistical significance $(\mathrm{P}<0.01$, Table 10). The thymic carcinoma tissue without staining was a negative control (Figure 27).

\section{Correlation between the expression of c-kit and EMT molecules}

The 22 cases of c-kit positive expression, 7 were E-cad positive, 13 were $\mathrm{N}$-cad positive, 15 were Twist positive, and 15 were Snail positive. Among the 103 cases with positive E-cad expression, 5 were $\mathrm{N}$-cad positive, 18 were Twist positive, and 14 were Snail positive. Among the $19 \mathrm{~N}$-cadpositive cases, 16 were Twist-positive, and 14 were Snailpositive. Among the 40 cases of Twist positive expression, 22 were Snail positive. There was a negative correlation between c-kit and E-cad expression (Table 11). As shown in Tables 1214, c-kit was positively correlated with the expression of $\mathrm{N}$-cad, Twist, and Snail while E-cad was negatively correlated with the expression of N-cad, Twist, and Snail (Tables 15-17). $\mathrm{N}$-cad was positively correlated with the expression of Twist and Snail $($ Table 18,19) while Twist was positively correlated with the expression of Snail (Table 20).

\section{Discussion}

TETs are derived from thymic epithelial cells and are composed of a series of histologically distinct tumors. In recent years, the International Thymic Malignancy 
Table 11 Relationship between the expression of c-kit and E-cad in thymic epithelial tumors

\begin{tabular}{|c|c|c|c|c|c|}
\hline \multirow{2}{*}{ c-kit } & \multirow{2}{*}{ Cases } & \multicolumn{2}{|c|}{ E-cad } & \multirow{2}{*}{$r$} & \multirow{2}{*}{$P$} \\
\hline & & + & - & & \\
\hline+ & 22 & 7 & 15 & -0.329 & $<0.05$ \\
\hline- & 128 & 96 & 32 & & \\
\hline
\end{tabular}

Table 12 Relationship between the expression of c-kit and N-cad in thymic epithelial tumors

\begin{tabular}{lrrrrrc}
\hline \multirow{2}{*}{ c-kit } & Cases & \multicolumn{3}{c}{ N-cad } & & r \\
\cline { 3 - 5 } & & + & & - & & \\
\hline+ & 22 & 13 & 9 & 0.579 & $<0.05$ \\
- & 128 & 6 & 122 & & \\
\hline
\end{tabular}

Table 13 Relationship between the expression of c-kit and Twist in thymic epithelial tumors

\begin{tabular}{|c|c|c|c|c|c|}
\hline \multirow{2}{*}{ c-kit } & \multirow{2}{*}{ Cases } & \multicolumn{2}{|c|}{ Twist } & \multirow{2}{*}{$r$} & \multirow{2}{*}{$\mathrm{P}$} \\
\hline & & + & - & & \\
\hline+ & 22 & 15 & 7 & 0.389 & $<0.05$ \\
\hline- & 128 & 25 & 103 & & \\
\hline
\end{tabular}

Table 14 Relationship between the expression of c-kit and Twist in thymic epithelial tumors

\begin{tabular}{|c|c|c|c|c|c|}
\hline \multirow{2}{*}{ c-kit } & \multirow{2}{*}{ Cases } & \multicolumn{2}{|c|}{ Snail } & \multirow{2}{*}{$r$} & \multirow{2}{*}{$P$} \\
\hline & & + & - & & \\
\hline+ & 22 & 15 & 7 & 0.451 & $<0.05$ \\
\hline- & 128 & 19 & 109 & & \\
\hline
\end{tabular}

Table 15 Relationship between the expression of E-cad and N-cad in thymic epithelial tumors

\begin{tabular}{|c|c|c|c|c|c|}
\hline \multirow{2}{*}{ E-cad } & \multirow{2}{*}{ Cases } & \multicolumn{2}{|c|}{$\mathrm{N}$-cad } & \multirow{2}{*}{$r$} & \multirow{2}{*}{$P$} \\
\hline & & + & - & & \\
\hline+ & 103 & 5 & 98 & -0.348 & $<0.05$ \\
\hline- & 47 & 14 & 33 & & \\
\hline
\end{tabular}

Table 16 Relationship between the expression of E-cad and Twist in thymic epithelial tumors

\begin{tabular}{|c|c|c|c|c|c|}
\hline \multirow{2}{*}{ E-cad } & \multirow{2}{*}{ Cases } & \multicolumn{2}{|c|}{ Twist } & \multirow{2}{*}{$r$} & \multirow{2}{*}{$P$} \\
\hline & & + & - & & \\
\hline+ & 103 & 18 & 85 & -0.308 & $<0.05$ \\
\hline - & 47 & 22 & 25 & & \\
\hline
\end{tabular}

Table 17 Relationship between the expression of E-cad and Snail in thymic epithelial tumors

\begin{tabular}{|c|c|c|c|c|c|}
\hline \multirow{2}{*}{ E-cad } & \multirow{2}{*}{ Cases } & \multicolumn{2}{|c|}{ Snail } & \multirow{2}{*}{$r$} & \multirow{2}{*}{$\mathrm{P}$} \\
\hline & & + & - & & \\
\hline+ & 103 & 14 & 89 & -0.321 & $<0.05$ \\
\hline - & 47 & 20 & 27 & & \\
\hline
\end{tabular}

Table 18 Relationship between the expression of $\mathrm{N}$-cad and Twist in thymic epithelial tumors

\begin{tabular}{|c|c|c|c|c|c|}
\hline \multirow{2}{*}{$\mathrm{N}$-cad } & \multirow{2}{*}{ Cases } & \multicolumn{2}{|c|}{ Twist } & \multirow{2}{*}{$r$} & \multirow{2}{*}{$\mathrm{P}$} \\
\hline & & + & - & & \\
\hline+ & 19 & 16 & 3 & 0.496 & $<0.05$ \\
\hline- & 131 & 24 & 107 & & \\
\hline
\end{tabular}

Table 19 Relationship between the expression of N-cad and Snail in thymic epithelial tumors

\begin{tabular}{|c|c|c|c|c|c|}
\hline \multirow{2}{*}{$\mathrm{N}$-cad } & \multirow{2}{*}{ Cases } & \multicolumn{2}{|c|}{ Snail } & \multirow{2}{*}{$r$} & \multirow{2}{*}{$P$} \\
\hline & & + & - & & \\
\hline+ & 19 & 14 & 5 & 0.444 & $<0.05$ \\
\hline- & 131 & 20 & 111 & & \\
\hline
\end{tabular}

Table 20 Relationship between the expression of Twist and Snail in thymic epithelial tumors

\begin{tabular}{|c|c|c|c|c|c|}
\hline \multirow{2}{*}{ Twist } & \multirow{2}{*}{ Cases } & \multicolumn{2}{|c|}{ Snail } & \multirow{2}{*}{$r$} & \multirow{2}{*}{$\mathrm{P}$} \\
\hline & & + & - & & \\
\hline+ & 40 & 22 & 18 & 0.466 & $<0.05$ \\
\hline- & 110 & 12 & 98 & & \\
\hline
\end{tabular}


Interest Group (ITG) proposed a formal and practical staging system. The Chinese Thymoma Cooperative Group has also been formed to conduct in-depth research and evaluate the prognosis factor of TETs (3). With the continuous advancement of tumor molecular research, molecular targeted therapy has been widely used in many clinical tumors. For TETs, there are also reports showed the application of molecular targeted drug therapy at home and abroad. Therefore, illuminating the molecular mechanism of thymic tumors not only helps to understand the occurrence, development, and metastasis of TETs but also points the way for the development of targeted drugs.

The current immunohistochemical results showed the statistically positive expression of c-kit receptors in each subtype (WHO classification) of the TET, especially in thymic carcinoma, which was significantly higher than that of thymoma and consistent with previous reports, suggesting that c-kit is associated with the malignancy of TETs.

EMT represents a cellular program that confers the tumor epithelial cells with the biological properties required for most of the invasion-metastasis cascade process. The progression of metastasis is a collection of different steps that make up the "invasion-transition cascade." First, cancer cells in the primary tumor acquire the ability to invade into surrounding tissues, which is necessary to break through the basement membrane that limits the epithelial space. The tumor cells then can make access to the lymphatic vessels and blood vessels, infiltrate, and transport in these lumens inactive form and finally extravasate. Finally, during colonization, small cell clumps, or individual tumortransmitting cells (micro-metastases) acquire the ability to survive and proliferate in different tissue microenvironments to form macroscopic metastases.

The immunohistochemistry results showed that there was a statistically significant difference in the positive expression of E-cadherin, $\mathrm{N}$-cadherin, Twist, and Snail in each subtype of TET. The expression level of E-cadherin, $\mathrm{N}$-cadherin, Twist, and Snail in thymoma was significantly higher than that in the thymus $(\mathrm{P}<0.01)$, consistent with the process of EMT in which the expression of E-cadherin was down-regulated, and the expression of $\mathrm{N}$-cadherin was up-regulated. Down-regulation of E-cadherin results in decreased or absent adhesion between tumor cells and tumor cells are easily isolated from the primary tumor, making it accessible to the invasive growth and metastasis.

The current study showed that the expression level of c-kit was negatively correlated with the expression of
E-cadherin and positively correlated with the expression of $\mathrm{N}$-cadherin, Twist, and Snail, suggesting that c-kit may be related to the occurrence and development of EMT. Tang et al. reported that c-kit could induce EMT to promote the progression of salivary adenoid cystic carcinoma, the main mechanism lies in the overexpression of c-kit in adenoid cystic carcinoma cell lines, and the TGF- $\beta$ signaling pathway is activated to promote the EMT process and also induce c-kit expression, further promoting the occurrence of EMT (39). Therefore, combined with our experimental results, overexpression of c-kit in TETs may also induce EMT processes. Correlation analysis showed that the expression of E-cadherin was negatively correlated with the expression of N-cadherin, Twist, and Snail. The expression of the transcription factors Twist and Snail inhibits the expression of E-cadherin by binding to the specific sequence of the E-cadherin promoter, thereby promoting the EMT process (40).

\section{Conclusions}

The expression of c-kit, E-cadherin, N-cadherin, Twist, and Snail in the TETs was significantly different, suggesting that these five indicators can determine the degree of benign and malignant TETs, especially for distinguishing between thymoma and thymic cancer. What is more, the expression of c-kit was negatively correlated with the expression of E-cadherin, and positively correlated with the expression of $\mathrm{N}$-cadherin, Twist, and Snail, suggesting that c-kit overexpression may promote EMT in TETs. The expression of Twist was positively correlated with the expression of Snail, suggesting that Twist and Snail may have synergistic effects, and the combined expression can promote tumor metastasis.

\section{Acknowledgments}

Funding: The study was financially supported by the Fujian Provincial Key Project (2014Y0024) and Fujian Provincial Joint Research Project of Health Care and Education (WKJ2016-2-09).

\section{Footnote}

Conflicts of Interest: The authors have no conflicts of interest to declare.

Ethical Statement: The authors are accountable for all 
aspects of the work in ensuring that questions related to the accuracy or integrity of any part of the work are appropriately investigated and resolved. The study protocol has been approved by the ethics committee on human research of Fujian Medical University. All patients gave their informed consents for participation and publication prior to their inclusion in the study.

\section{References}

1. Travis WD, Brambilla E, Burke AP, et al. Introduction to The 2015 World Health Organization Classification of Tumors of the Lung, Pleura, Thymus, and Heart. J Thorac Oncol 2015;10:1240-2.

2. Marx A, Strobel P, Badve SS, et al. ITMIG consensus statement on the use of the WHO histological classification of thymoma and thymic carcinoma: refined definitions, histological criteria, and reporting. J Thorac Oncol 2014;9:596-611.

3. Detterbeck F, Youssef S, Ruffini E, et al. A review of prognostic factors in thymic malignancies. J Thorac Oncol 2011;6:S1698-1704.

4. Besmer P, Murphy JE, George PC, et al. A new acute transforming feline retrovirus and relationship of its oncogene $v$-kit with the protein kinase gene family. Nature 1986;320:415-21.

5. Buti S, Donini M, Sergio $P$, et al. Impressive response with imatinib in a heavily pretreated patient with metastatic c-KIT mutated thymic carcinoma. J Clin Oncol 2011;29:e803-805.

6. Giaccone G, Rajan A, Ruijter R, et al. Imatinib mesylate in patients with WHO B3 thymomas and thymic carcinomas. J Thorac Oncol 2009;4:1270-3.

7. Palmieri G, Marino M, Buonerba C, et al. Imatinib mesylate in thymic epithelial malignancies. Cancer Chemother Pharmacol 2012;69:309-15.

8. Pan CC, Chen PC, Chiang H. Overexpression of KIT (CD117) in chromophobe renal cell carcinoma and renal oncocytoma. Am J Clin Pathol 2004;121:878-83.

9. Corless CL, Fletcher JA, Heinrich MC. Biology of gastrointestinal stromal tumors. J Clin Oncol 2004:22:3813-25.

10. Inokuchi K, Yamaguchi H, Tarusawa M, et al. Abnormality of c-kit oncoprotein in certain patients with chronic myelogenous leukemia--potential clinical significance. Leukemia 2002;16:170-7.

11. Nakagawa K, Matsuno Y, Kunitoh H, et al. Immunohistochemical KIT (CD117) expression in thymic epithelial tumors. Chest 2005;128:140-4.

12. Yoh K, Nishiwaki Y, Ishii G, et al. Mutational status of EGFR and KIT in thymoma and thymic carcinoma. Lung Cancer 2008;62:316-20.

13. Greenburg G, Hay ED. Epithelia suspended in collagen gels can lose polarity and express characteristics of migrating mesenchymal cells. J Cell Biol 1982;95:333-9.

14. Nickel A, Stadler SC. Role of epigenetic mechanisms in epithelial-to-mesenchymal transition of breast cancer cells. Transl Res 2015;165:126-42.

15. Liu H, Yin J, Wang H, et al. FOXO3a modulates WNT/ beta-catenin signaling and suppresses epithelial-tomesenchymal transition in prostate cancer cells. Cell Signal 2015;27:510-8.

16. Kalluri R, Weinberg RA. The basics of epithelialmesenchymal transition. J Clin Invest 2009;119:1420-8.

17. Lamouille S, Xu J, Derynck R. Molecular mechanisms of epithelial-mesenchymal transition. Nat Rev Mol Cell Biol 2014;15:178-96.

18. Zhang H, Liu L, Wang Y, et al. KLF8 involves in TGF-beta-induced EMT and promotes invasion and migration in gastric cancer cells. J Cancer Res Clin Oncol 2013;139:1033-42.

19. Vittal R, Fan L, Greenspan DS, et al. IL-17 induces type V collagen overexpression and EMT via TGF-betadependent pathways in obliterative bronchiolitis. Am J Physiol Lung Cell Mol Physiol 2013;304:L401-414.

20. Massagué J. TGFbeta in Cancer. Cell 2008;134:215-30.

21. Shi Y, Massague J. Mechanisms of TGF-beta signaling from cell membrane to the nucleus. Cell 2003;113:685-700.

22. Elloul S, Elstrand MB, Nesland JM, et al. Snail, Slug, and Smad-interacting protein 1 as novel parameters of disease aggressiveness in metastatic ovarian and breast carcinoma. Cancer 2005;103:1631-43.

23. Thiery JP, Huang R. Linking epithelial-mesenchymal transition to the well-known polarity protein Par6. Dev Cell 2005;8:456-8.

24. Singh A, Settleman J. EMT, cancer stem cells and drug resistance: an emerging axis of evil in the war on cancer. Oncogene 2010;29:4741-51.

25. Hazan RB, Qiao R, Keren R, et al. Cadherin switch in tumor progression. Ann N Y Acad Sci 2004;1014:155-63.

26. Toivola DM, Tao GZ, Habtezion A, et al. Cellular integrity plus: organelle-related and protein-targeting functions of intermediate filaments. Trends Cell Biol 2005;15:608-17.

27. Lorenz KJ, Kraft K, Graf F, et al. Role of reflux-induced epithelial-mesenchymal transition in periprosthetic leakage after prosthetic voice rehabilitation. Head Neck 
2015;37:530-6.

28. Hazan RB, Phillips GR, Qiao RF, et al. Exogenous expression of $\mathrm{N}$-cadherin in breast cancer cells induces cell migration, invasion, and metastasis. J Cell Biol 2000;148:779-90.

29. Li G, Satyamoorthy K, Herlyn M. N-cadherin-mediated intercellular interactions promote survival and migration of melanoma cells. Cancer Res 2001;61:3819-25.

30. Derycke LD, Bracke ME. N-cadherin in the spotlight of cell-cell adhesion, differentiation, embryogenesis, invasion and signalling. Int J Dev Biol 2004;48:463-76.

31. Gwak GY, Yoon JH, Yu SJ, et al. Anti-apoptotic $\mathrm{N}$-cadherin signaling and its prognostic implication in human hepatocellular carcinomas. Oncol Rep 2006;15:1117-23.

32. Horvai AE, Roy R, Borys D, et al. Regulators of skeletal development: a cluster analysis of 206 bone tumors reveals diagnostically useful markers. Mod Pathol 2012;25:1452-61.

33. Lee MS, Lowe G, Flanagan S, et al. Human Dermo-1 has attributes similar to twist in early bone development. Bone 2000;27:591-602.

Cite this article as: $\mathrm{Wu} \mathrm{Z}$, Xue S, Zheng B, Ye R, Xu G, Zhang S, Zeng T, Zheng W, Chen C. Expression and significance of c-kit and epithelial-mesenchymal transition (EMT) molecules in thymic epithelial tumors (TETs). J Thorac Dis 2019;11(11):4602-4612. doi: 10.21037/jtd.2019.10.56
34. Yang J, Mani SA, Donaher JL, et al. Twist, a master regulator of morphogenesis, plays an essential role in tumor metastasis. Cell 2004;117:927-39.

35. Sahlin $\mathrm{P}$, Windh $\mathrm{P}$, Lauritzen $\mathrm{C}$, et al. Women with Saethre-Chotzen syndrome are at increased risk of breast cancer. Genes Chromosomes Cancer 2007;46:656-60.

36. Khan MA, Chen HC, Zhang D, et al. Twist: a molecular target in cancer therapeutics. Tumour Biol 2013;34:2497-506.

37. Wang Y, Shi J, Chai K, et al. The Role of Snail in EMT and Tumorigenesis. Curr Cancer Drug Targets 2013;13:963-72.

38. Barrallo-Gimeno A, Nieto MA. The Snail genes as inducers of cell movement and survival: implications in development and cancer. Development 2005;132:3151-61.

39. Tang YL, Fan YL, Jiang J, et al. C-kit induces epithelialmesenchymal transition and contributes to salivary adenoid cystic cancer progression. Oncotarget 2014;5:1491-501.

40. van Nes JG, de Kruijf EM, Putter H, et al. Co-expression of SNAIL and TWIST determines prognosis in estrogen receptor-positive early breast cancer patients. Breast Cancer Res Treat 2012;133:49-59. 